\title{
Preface for the special volume on Computational Intelligence in Scheduling
}

\author{
Graham Kendall · Kay Chen Tan • Edmund Burke • \\ Stephen Smith
}

Published online: 15 July 2009

(C) Springer Science+Business Media, LLC 2009

This special volume of Annals of Operations Research contains a collection of papers arising from the 2007 IEEE Symposium on Computational Intelligence in Scheduling, April 1-5, 2007, Honolulu, Hawaii, USA.

The eleven papers in this special volume underwent a full review process, as would be expected from a high quality international journal.

We have presented the papers in three sections, these being:

1) Real World Applications

2) Job and Machine Scheduling

3) Theoretical Issues

\section{Section 1: Real World Applications}

This section contains five papers. Mumford presents a multi-objective framework which is used to solve heavily constrained timetabling problems. Results are most promising for capacitated problems (i.e., those in which room capacities have to be respected) and those problems in which some examinations have a restricted set of timeslots. Lamghari and Ferland assign judges to tournaments by utilizing two meta-heuristic methods, both of which are based on tabu search. The proposed methodology is tested on the John Molson International Case Competition, which has taken place at Concordia University in Montreal (Canada) for

G. Kendall $(\bowtie) \cdot$ E. Burke

University of Nottingham, Nottingham, UK

e-mail: gxk@cs.nott.ac.uk

K.C. Tan

National University of Singapore, Singapore, Singapore

S. Smith

Carnegie Mellon University, Pittsburgh, USA 
the last 25 years. Very good solutions are reported. Cheong, Tan, Liu, and Lin consider the problem of determining the berthing times and positions of ships arriving at a container port. The problem is modelled as a multi-objective problem (the objectives being makespan, waiting time and deviation from the schedule). This multi-objective approach presents the user with a Pareto front of solutions from which to choose. Fadiloĝlu, Karaşan, and Pinar present a model that yields the optimal set of products to be placed on a shelf to achieve the maximum profit. The model is applied to two real world datasets from local supermarket chains. Oliveira and Vasconcelos combine simulated annealing, hill climbing, and random restart, and apply their proposed methodology to the vehicle routing problem with time windows. They improve on many of the results from the 56 instances in the Solomon dataset.

\section{Section 2: Job and Machine Scheduling}

This section contains four papers. Franke, Lepping, and Schwiegelshohn consider a scheduling problem comprising independent parallel jobs and parallel identical machines. Their proposed algorithm combines a greedy search with a dynamic sorting of the waiting queue. They evaluate their proposed methodology on real workload data, comparing it to an optimal offline strategy as well as the EASY backfill algorithm. Grobler, Engelbrecht, Kok, and Yadavalli investigate four different particle swarm optimization algorithms for a multiobjective flexible job shop scheduling problem. Their results show that their algorithms are superior to other methodologies when there are 125 or less operations to be scheduled. Chang, Chen, Fan, and Mani propose a novel genetic algorithm that draws its inspiration from evolutionary algorithms based on probabilistic models in order to solve machine scheduling problems. They show that the proposed algorithm is superior to some other algorithms from the literature. Shenfield, Fleming, Kadirkamanathan, and Allan introduce a Grid-based optimization framework. The framework is used to optimize maintenance scheduling strategies for aero-engines, which results in substantial savings in terms of computational time.

\section{Section 3: Theoretical Issues}

This section contains two papers. Barták, Čepek, and Surynek study three methods to improve constraint reasoning in P/A graphs (an extension of precedence graphs). The research is motivated by the observation that some information known to the user is often lost in the formal model, and the proposed method attempts to rediscover lost information. Kuster, Jannach, and Friedrich present an approach to manage scheduling disruptions. The method reduces the size of the search space by applying partial scheduling and the technique is shown to perform very well, particularly on large, complex problems.

Finally, we would like to thank all those who attended the symposium and all those who submitted their papers to this special volume. In particular, we would like to thank all the reviewers. Without their help, support, expertise, and patience this volume would not have been possible. 